\title{
DIMENSION FREE BOUNDEDNESS OF RIESZ TRANSFORMS FOR THE GRUSHIN OPERATOR
}

\author{
P. K. SANJAY AND S. THANGAVELU \\ (Communicated by Alexander Iosevich)
}

Abstract. Let $G=-\Delta_{\xi}-|\xi|^{2} \frac{\partial^{2}}{\partial \eta^{2}}$ be the Grushin operator on $\mathbb{R}^{n} \times \mathbb{R}$. We prove that the Riesz transforms associated to this operator are bounded on $L^{p}\left(\mathbb{R}^{n+1}\right), 1<p<\infty$, and their norms are independent of dimension $n$.

\section{INTRODUCTION}

We consider the Grushin operator $G=-\Delta_{\xi}-|\xi|^{2} \frac{\partial^{2}}{\partial \eta^{2}}$ on $\mathbb{R}^{n} \times \mathbb{R}$ which can be written formally as

$$
G f(\xi, \eta)=\frac{1}{2 \pi} \int_{\mathbb{R}} e^{-i \lambda \eta} H(\lambda) f^{\lambda}(\xi) d \lambda
$$

where

$$
H(\lambda)=-\Delta+\lambda^{2}|\xi|^{2}
$$

is the scaled Hermite operator on $\mathbb{R}^{n}$ and

$$
f^{\lambda}(\xi)=\int_{\mathbb{R}} f(\xi, \eta) e^{i \lambda \eta} d \eta
$$

is the inverse Fourier transform of $f$ in the $\eta$ variable. In the light of this decomposition, we can define the Riesz transforms associated to the operator $G$ as

$$
R_{j} f(\xi, \eta)=\int_{-\infty}^{\infty} e^{-i \lambda \eta} R_{j}(\lambda) f^{\lambda}(\xi) d \lambda
$$

and

$$
R_{j}^{*} f(\xi, \eta)=\int_{-\infty}^{\infty} e^{-i \lambda \eta} R_{j}^{*}(\lambda) f^{\lambda}(\xi) d \lambda
$$

for $j=1,2,3, \ldots, n$. Here,

$$
R_{j}(\lambda)=A_{j}(\lambda) H(\lambda)^{-\frac{1}{2}}, \quad R_{j}^{*}(\lambda)=A_{j}(\lambda)^{*} H(\lambda)^{-\frac{1}{2}}
$$

are the Riesz transforms associated to the Hermite operator which has the decomposition

$$
H(\lambda)=\frac{1}{2} \sum_{j=1}^{n}\left(A_{j}(\lambda) A_{j}(\lambda)^{*}+A_{j}(\lambda)^{*} A_{j}(\lambda)\right)
$$

where

$$
A_{j}(\lambda)=-\frac{\partial}{\partial \xi_{j}}+\lambda \xi_{j}, \quad A_{j}(\lambda)^{*}=\frac{\partial}{\partial \xi_{j}}+\lambda \xi_{j}
$$

Received by the editors August 11, 2012 and, in revised form, November 17, 2012. 2010 Mathematics Subject Classification. Primary 42Cxx, 42C05, 43A65. 
are the creation and annihilation operators on $\mathbb{R}^{n}$. The boundedness of these Riesz transforms on $L^{p}\left(\mathbb{R}^{n+1}\right)$ is known [JST, BG]. In [BG], the authors have proved the boundedness of Riesz transforms associated to a much larger class of smooth locally subelliptic diffusion operators on a smooth connected non-compact manifold. In [JST these Riesz transforms were treated as operator valued Fourier multipliers for $L^{p}\left(\mathbb{R}^{n}\right)$ valued functions on $\mathbb{R}$ and the boundedness was proved with the aid of a result of L. Weis Wei01, on the operator valued Fourier multipliers. The aim of this paper is to prove the following theorem about the dimension free boundedness of the vector of Riesz transforms $\mathcal{R} f$. That is, we consider the operator $\mathcal{R}=$ $\left(R_{1}, R_{2}, \cdots, R_{n}, R_{1}^{*}, R_{2}^{*}, \cdots, R_{n}^{*}\right)$ with

$$
|\mathcal{R} f(\xi, \eta)|=\left(\sum_{j=1}^{n}\left|R_{j} f(\xi, \eta)\right|^{2}+\sum_{j=1}^{n}\left|R_{j}^{*} f(\xi, \eta)\right|^{2}\right)^{1 / 2}
$$

and prove:

Theorem 1.1. For each $1<p<\infty$, there exists a constant $C_{p}$ independent of dimension $n$ such that for all $f \in L^{p}\left(\mathbb{R}^{n} \times \mathbb{R}\right)$,

$$
\|\mathcal{R} f\|_{p} \leq C_{p}\|f\|_{p}
$$

E. M. Stein introduced the notion of the dimension free boundedness for the vector of Riesz transforms in Ste83. Stein considered the Euclidean Riesz transforms and proved the result using the technique of g-functions. Later this technique was adapted for a non-commutative situation by Harboure et al. [HRST04] to prove a similar result for the Riesz transforms associated to the Hermite operator. Alternate proofs for the Euclidean Riesz transforms were provided using the method of rotations by J. Duoandikoetxea and J. L. Rubio de Francia [DR85] and using a transference argument by Gilles Pisier Pis88. Similar techniques have been used to prove the dimension free boundedness of Riesz transforms in other contexts as well. Coulhon, Müller and Zienkiewicz [CMZ96] proved the boundedness for the Riesz transforms associated to the sub-Laplacian on the Heisenberg group. In two works, [LP06] and [LP04], Francoise Lust-Piquard provided alternate proofs for the Riesz transforms associated to the Hermite operator and the Riesz transforms associated to the sub-Laplacian. Our proof follows a method very similar to that of [CMZ96], [LP04] and [LP06]. The method of rotations applied to the Euclidean Riesz transforms DR85] involves expressing the Riesz transforms as the average of certain directional Hilbert transforms and using the boundedness (independent of the direction) of these directional Hilbert transforms. An expression similar to this in the Heisenberg group context is obtained in [CMZ96. In this paper we obtain a similar representation for Riesz transforms associated to Grushin operators.

We also note that Theorem 1.1 in turn implies that the Riesz transforms associated to the Hermite operator satisfy dimension free bounds. Since the Riesz transform for the Grushin operator is an operator valued multiplier for the Fourier transform on $\mathbb{R}$, the corresponding multiplier operator for the Fourier series is also bounded by the same norm. This can be proved using a generalisation of a transference result of Karel de Leeuw dL65. The proof is similar to the proof in [ST12] of the boundedness of the Riesz transforms on the reduced Heisenberg group using transference from the Heisenberg group. We refer to Theorem 2.1 and Theorem 2.2 
of ST12 for details. Then the proof of the Hermite Riesz transforms follows by looking at functions of the form $F(\xi, \eta)=f(\xi) e^{i k \eta}$ on $\mathbb{R}^{n} \times[0,2 \pi)$.

\section{Riesz transforms FOR the GRUShin operator}

We first consider the individual Riesz transforms $R_{j}$ and $R_{j}^{*}$ for $j=1,2, \cdots, n$ and show that they satisfy dimension free bounds on $L^{p}\left(\mathbb{R}^{n+1}\right)$ for $1<p<\infty$. In order to do this we introduce the operators $R_{j}^{\epsilon}$ and $R_{j}^{* \epsilon}$, which we will call the truncated Riesz transforms. We only give details of $R_{j}^{\epsilon}$, as the other one is similar. Note that the Riesz transform $R_{j}(\lambda)$ associated to the Hermite operator $H(\lambda)$ can be written as

$$
R_{j}(\lambda)=A_{j}(\lambda) H(\lambda)^{-1 / 2}=\frac{A_{j}(\lambda)}{\sqrt{\pi}} \int_{0}^{\infty} e^{-r H(\lambda)} r^{-1 / 2} d r .
$$

Here $e^{-r H(\lambda)}$ is the Hermite semigroup. For $\epsilon>0$, we define the truncated Riesz transforms $R_{j}^{\epsilon}(\lambda)$ by

$$
R_{j}^{\epsilon}(\lambda)=\frac{A_{j}(\lambda)}{\sqrt{\pi}} \int_{\epsilon^{2}}^{1 / \epsilon^{2}} e^{-r H(\lambda)} r^{-1 / 2} d r .
$$

Then the truncated Riesz transforms $R_{j}^{\epsilon}$ for the Grushin operator are defined as

$$
R_{j}^{\epsilon} f(\xi, \eta)=\frac{1}{2 \pi} \int_{-\infty}^{\infty} e^{-i \lambda \eta} R_{j}^{\epsilon}(\lambda) f^{\lambda}(\xi) d \lambda
$$

We first prove

Proposition 2.1. For every $f \in L^{2}\left(\mathbb{R}^{n+1}\right), R_{j}^{\epsilon} f \rightarrow R_{j} f$ in $L^{2}\left(\mathbb{R}^{n+1}\right)$ as $\epsilon \rightarrow 0$.

Proof. It follows from the definition that

$\int_{\mathbb{R}} \int_{\mathbb{R}^{n}}\left|R_{j}^{\epsilon} f(\xi, \eta)-R_{j} f(\xi, \eta)\right|^{2} d \xi d \eta=\int_{\mathbb{R}}\left(\int_{\mathbb{R}^{n}}\left|R_{j}^{\epsilon}(\lambda) f^{\lambda}(\xi)-R_{j}(\lambda) f^{\lambda}(\xi)\right|^{2} d \xi\right) d \lambda$.

The proposition follows once we show that for every $\lambda \in \mathbb{R}^{*}$

$$
\int_{\mathbb{R}^{n}}\left|R_{j}^{\epsilon}(\lambda) f^{\lambda}(\xi)-R_{j}(\lambda) f^{\lambda}(\xi)\right|^{2} d \xi \rightarrow 0 \text { as } \epsilon \rightarrow 0
$$

and

$$
\int_{\mathbb{R}^{n}}\left|R_{j}^{\epsilon}(\lambda) f^{\lambda}(\xi)-R_{j}(\lambda) f^{\lambda}(\xi)\right|^{2} d \xi \leq 4 \int_{\mathbb{R}^{n}}\left|f^{\lambda}(\xi)\right|^{2} d \xi .
$$

To see these, we expand $f^{\lambda}$ in terms of scaled Hermite functions $\Phi_{\alpha}^{\lambda}$ (see [Tha04] for a definition) and use the fact that

$$
A_{j}(\lambda) \Phi_{\alpha}^{\lambda}=\left(2 \alpha_{j}+2\right)^{1 / 2}|\lambda|^{1 / 2} \Phi_{\alpha+e_{j}}^{\lambda},
$$

where $e_{j}$ is the canonical unit vector of $\mathbb{R}^{n}$ with 1 in the $j^{\text {th }}$ entry and zero elsewhere. Then

$$
\begin{aligned}
R_{j}^{\epsilon}(\lambda) & f^{\lambda}(\xi)-R_{j}(\lambda) f^{\lambda}(\xi) \\
& =\sum_{\alpha \in \mathbb{N}^{n}} \frac{\left(2 \alpha_{j}+2\right)^{1 / 2}|\lambda|^{1 / 2}}{\sqrt{\pi}}\left(\int_{A_{\epsilon}} e^{-(2|\alpha|+n)|\lambda| r} r^{-1 / 2} d r\right)\left(f^{\lambda}, \Phi_{\alpha}^{\lambda}\right) \Phi_{\alpha+e_{j}}^{\lambda}(\xi),
\end{aligned}
$$


where $A_{\epsilon}=\left(0, \epsilon^{2}\right) \cup\left(1 / \epsilon^{2}, \infty\right)$. From this, it follows that

$$
\left\|R_{j}^{\epsilon}(\lambda) f^{\lambda}-R_{j}(\lambda) f^{\lambda}\right\|_{2}^{2}=\frac{1}{\pi} \sum_{\alpha \in \mathbb{N}^{n}} \frac{2 \alpha_{j}+2}{2|\alpha|+n}\left|\left(f^{\lambda}, \Phi_{\alpha}^{\lambda}\right)\right|^{2}\left(\int_{A_{\epsilon, \alpha}} e^{-r} r^{-1 / 2} d r\right)^{2},
$$

where $A_{\epsilon, \alpha}=\left(0,(2|\alpha|+n)|\lambda| \epsilon^{2}\right) \cup\left((2|\alpha|+n)|\lambda| \epsilon^{-2}, \infty\right)$. From the above equation it is clear that

$$
\left\|R_{j}^{\epsilon}(\lambda) f^{\lambda}-R_{j}(\lambda) f^{\lambda}\right\|_{2} \leq 2\left\|f^{\lambda}\right\|_{2} .
$$

We also note that when

$$
f^{\lambda}(\xi)=\sum_{(2|\alpha|+n)|\lambda| \leq N}\left(f^{\lambda}, \Phi_{\alpha}^{\lambda}\right) \Phi_{\alpha}^{\lambda}(\xi)
$$

is a finite linear combination of $\Phi_{\alpha}^{\lambda}$,

$$
\left\|R_{j}^{\epsilon}(\lambda) f^{\lambda}-R_{j}(\lambda) f^{\lambda}\right\|_{2}^{2} \leq \frac{1}{\pi} \sum_{(2|\alpha|+n)|\lambda| \leq N}\left|\left(f^{\lambda}, \Phi_{\alpha}^{\lambda}\right)\right|^{2}\left(\int_{\left(0, N \epsilon^{2}\right) \cup\left(N \epsilon^{-2}, \infty\right)} e^{-r} r^{-1 / 2} d r\right)^{2},
$$

which goes to 0 as $\epsilon \rightarrow 0$. As such functions are dense in $L^{2}\left(\mathbb{R}^{n}\right)$, we get

$$
\left\|R_{j}^{\epsilon}(\lambda) f^{\lambda}-R_{j}(\lambda) f^{\lambda}\right\|_{2} \rightarrow 0, \text { as } \epsilon \rightarrow 0,
$$

for any $f \in L^{2}\left(\mathbb{R}^{n+1}\right)$.

For the individual Riesz transforms, we have the following result:

Theorem 2.2. For $j=1,2,3, \cdots, n$ we have

$$
\left\|R_{j} f\right\|_{p}+\left\|R_{j}^{*} f\right\|_{p} \leq C_{p}\|f\|_{p}, \quad 1<p<\infty
$$

for all $f \in L^{p}\left(\mathbb{R}^{n+1}\right)$, where $C_{p}$ is independent of the dimension.

In order to prove this result, we claim that it is enough to prove

$$
\left\|R_{j}^{\epsilon} f\right\|_{p}+\left\|R_{j}^{* \epsilon} f\right\|_{p} \leq C_{p}\|f\|_{p}
$$

for the truncated Riesz transforms. A proof of this will be given in section 5 . where we will show that $\left(R_{j}^{\epsilon} f\right)$ is Cauchy in $L^{p}\left(\mathbb{R}^{n+1}\right)$ and hence there exists an operator $S_{j}$, bounded on $L^{p}\left(\mathbb{R}^{n+1}\right)$ such that $R_{j}^{\epsilon} f \rightarrow S_{j} f$ as $\epsilon \rightarrow 0$. In view of Proposition 2.1, $R_{j}^{\epsilon} f \rightarrow R_{j} f$ for $f \in L^{2}\left(\mathbb{R}^{n+1}\right)$, and hence $S_{j} f=R_{j} f$. This will prove the stated boundedness of $R_{j}$ (and $R_{j}^{*}$ ). Now to prove the boundedness of $R_{j}^{\epsilon}$ and $R_{j}^{* \epsilon}$ we express these operators as a superposition of certain truncated Hilbert transforms.

\section{A Representation For the truncated Riesz transforms}

The representation we obtain is very similar to the representation obtained in CMZ96] for the Riesz transforms on the Heisenberg group $\mathbb{H}^{n}$. Before stating this result, we recall some definitions and notation. For further details and proofs we refer to [Tha04.

Recall that as a manifold $\mathbb{H}^{n}=\mathbb{C}^{n} \times \mathbb{R}$, and hence we write $(z, t), z=x+i y \in$ $\mathbb{C}^{n}, t \in \mathbb{R}$, to denote the elements of $\mathbb{H}^{n}$. The sub-Laplacian $\mathcal{L}$ on the Heisenberg group $\mathbb{H}^{n}$ can be written in terms of the differential operators $X_{j}=\left(\frac{\partial}{\partial x_{j}}+\frac{1}{2} y_{j} \frac{\partial}{\partial t}\right)$ 
and $Y_{j}=\left(\frac{\partial}{\partial y_{j}}-\frac{1}{2} x_{j} \frac{\partial}{\partial t}\right)$ as $\mathcal{L}=\sum_{j=1}^{n}\left(X_{j}^{2}+Y_{j}^{2}\right)$. The sub-Laplacian is homogeneous of degree 2 with respect to the non-isotropic dilations $\delta_{r}(z, t)=\left(r z, r^{2} t\right)$ of the Heisenberg group. Hence $p_{s}(z, t)$, the heat kernel associated to $\mathcal{L}$, satisfies

$$
p_{r^{2} s}(z, t)=r^{-(2 n+2)} p_{s}\left(z / r, t / r^{2}\right) .
$$

This also follows from the following explicit expression for $q_{s}(z, \lambda)$, the inverse Fourier transform of $p_{s}(z, t)$ in the $t$ variable:

$$
q_{s}(z, \lambda)=\int_{\mathbb{R}} p_{s}(z, t) e^{i \lambda t} d t=(4 \pi)^{-n}\left(\frac{\lambda}{\sinh \lambda s}\right)^{n} e^{-\frac{1}{4} \lambda \operatorname{coth}(s \lambda)|z|^{2}} .
$$

By $\pi_{\lambda}$, we denote the Schrödinger representation of the Heisenberg group $\mathbb{H}^{n}$ acting on $L^{2}\left(\mathbb{R}^{n}\right)$ in the following manner:

$$
\pi_{\lambda}(x+i y, t) \phi(\xi)=e^{i \lambda t} e^{i \lambda\left(x \cdot \xi+\frac{1}{2} x \cdot y\right)} \phi(\xi+y) .
$$

When $t=0$, we will denote $\pi_{\lambda}(z, t)$ by $\pi_{\lambda}(z) . \pi_{\lambda}$ also defines a representation of the group algebra $L^{1}\left(\mathbb{H}^{n}\right)$ as

$$
\pi_{\lambda}(f)=\int_{\mathbb{H}^{n}} f(z, t) \pi_{\lambda}(z, t) d z d t=W_{\lambda}\left(f^{\lambda}\right),
$$

where $W_{\lambda}(g)=\int_{\mathbb{C}^{n}} g(z) \pi_{\lambda}(z) d z$ is the Weyl transform of $g$. The representation given in CMZ96] for the Riesz transforms associated to the sub-Laplacian $\mathcal{L}$ is

$$
\left(X_{i} \mathcal{L}^{-\frac{1}{2}} f\right)(z, t)=-\frac{1}{4(2 \pi)^{n+1}} \int_{\mathbb{H}^{n}} X_{i} p_{1}(w, s) H_{(w, s)} f(z, t) d w d s,
$$

where $H_{(w, s)}$ is the Hilbert transform along a curve in the Heisenberg group. For the Grushin operator, we obtain a similar representation involving certain operators $T_{\epsilon}^{(z, t)}$ and certain differential operators

$$
\tilde{Z}_{j}=i \tilde{X}_{j}+\tilde{Y}_{j} ; \tilde{Z}_{j}^{*}=i \tilde{X}_{j}-\tilde{Y}_{j}
$$

with

$$
\tilde{X}_{j}=\left(\frac{\partial}{\partial x_{j}}-\frac{y_{j}}{2} \frac{\partial}{\partial t}\right) ; \tilde{Y}_{j}=\left(\frac{\partial}{\partial y_{j}}+\frac{x_{j}}{2} \frac{\partial}{\partial t}\right) .
$$

Proposition 3.1.

$$
R_{j}^{\epsilon} f(\xi, \eta)=\frac{1}{\sqrt{\pi}} \int_{\mathbb{H}^{n}} T_{\epsilon}^{(z, t)} f(\xi, \eta) \tilde{Z}_{j} p_{1}(z, t) d z d t
$$

and

where

$$
R_{j}^{* \epsilon} f(\xi, \eta)=\frac{1}{\sqrt{\pi}} \int_{\mathbb{H}^{n}} T_{\epsilon}^{(z, t)} f(\xi, \eta) \tilde{Z}_{j}^{*} p_{1}(z, t) d z d t
$$

$$
T_{\epsilon}^{(z, t)} f(\xi, \eta)=\int_{\epsilon<|r|<1 / \epsilon} f\left(\xi+r y, \eta+r x \cdot \xi+r^{2}\left(t+\frac{x \cdot y}{2}\right)\right) \frac{d r}{r} .
$$

We follow the method in [LP06] for proving this proposition. First we prove the following lemma, which will be used in the proof of Proposition 3.1 .

Lemma 3.2. For any $\phi \in \mathcal{S}\left(\mathbb{R}^{n}\right)$,

(i) $r \frac{\partial}{\partial \xi_{j}}\left(\pi_{\lambda}(r z) \phi(\xi)\right)=\left(\frac{\partial}{\partial y_{j}}+\frac{i \lambda r^{2} x_{j}}{2}\right)\left(\pi_{\lambda}(r z) \phi(\xi)\right)$,

(ii) $i r \lambda \xi_{j} \pi_{\lambda}(r z) \phi(\xi)=\left(\frac{\partial}{\partial x_{j}}-\frac{i \lambda r^{2} y_{j}}{2}\right) \pi_{\lambda}(r z) \phi(\xi)$. 
Proof. We first look at the derivative of $\pi_{\lambda}(r z)$ with respect to the variable $y_{j}$ and see that

$$
\begin{aligned}
\frac{\partial}{\partial y_{j}}\left(\pi_{\lambda}(r z) \phi(\xi)\right) & =\frac{\partial}{\partial y_{j}}\left(e^{i \lambda\left(r x \cdot \xi+\frac{1}{2} r^{2} x \cdot y\right)} \phi(\xi+r y)\right) \\
& =\frac{i \lambda r^{2} x_{j}}{2} \pi_{\lambda}(r z) \phi(\xi)+e^{i \lambda\left(r x \cdot \xi+\frac{1}{2} r^{2} x \cdot y\right)} \frac{\partial}{\partial y_{j}} \phi(\xi+r y) \\
& =\frac{i \lambda r^{2} x_{j}}{2} \pi_{\lambda}(r z) \phi(\xi)+r \pi_{\lambda}(r z) \frac{\partial \phi}{\partial \xi_{j}}(\xi) .
\end{aligned}
$$

Writing

$$
\pi_{\lambda}(r z) \frac{\partial \phi}{\partial \xi_{j}}(\xi)=\left(\pi_{\lambda}(r z) \frac{\partial}{\partial \xi_{j}}-\frac{\partial}{\partial \xi_{j}} \pi_{\lambda}(r z)\right) \phi(\xi)+\frac{\partial}{\partial \xi_{j}} \pi_{\lambda}(r z) \phi(\xi)
$$

and noting that

$$
\frac{\partial}{\partial \xi_{j}}\left(\pi_{\lambda}(r z) \phi(\xi)\right)=i \lambda r x_{j} \pi_{\lambda}(r z) \phi(\xi)+\pi_{\lambda}(r z) \frac{\partial \phi}{\partial \xi_{j}}(\xi),
$$

we see that

$$
\frac{\partial}{\partial y_{j}}\left(\pi_{\lambda}(r z) \phi(\xi)\right)=\left(r \frac{\partial}{\partial \xi_{j}}-\frac{i \lambda r^{2} x_{j}}{2}\right)\left(\pi_{\lambda}(r z) \phi(\xi)\right)
$$

which proves part (i). Part (ii) of the lemma follows from

$$
\frac{\partial}{\partial x_{j}}\left(\pi_{\lambda}(r z) \phi(\xi)\right)=i \lambda r \xi_{j} \pi_{\lambda}(r z) \phi(\xi)+\frac{i \lambda r^{2} y_{j}}{2} \pi_{\lambda}(r z) \phi(\xi) .
$$

Proof of Proposition 3.1. The heat kernels for the sub-Laplacian and the Hermite operators are related via the group Fourier transform on the Heisenberg group as follows:

$$
\hat{p_{s}}(\lambda)=\int_{\mathbb{H}^{n}} p_{s}(z, t) \pi_{\lambda}(z, t) d z d t=e^{-s H(\lambda)} .
$$

We refer to Tha04 for a proof of this. Using the homogeneity of the heat kernel we get

$$
\begin{aligned}
e^{-r^{2} H(\lambda)} & =r^{-(2 n+2)} \int_{\mathbb{H}^{n}} \pi_{\lambda}(z, t) p_{1}\left(\frac{z}{r}, \frac{t}{r^{2}}\right) d z d t \\
& =r^{-2 n} \int_{\mathbb{C}^{n}} \pi_{\lambda}(z) q_{1}\left(\frac{z}{r},-\lambda r^{2}\right) d z=\int_{\mathbb{C}^{n}} \pi_{\lambda}(r z) q_{1}\left(z,-\lambda r^{2}\right) d z .
\end{aligned}
$$

Since $R_{j}^{\epsilon}(\lambda)=\frac{1}{\sqrt{\pi}} A_{j}(\lambda) \int_{\epsilon<|r|<1 / \epsilon} e^{-r^{2} H(\lambda)} d r$, we see that

$$
\begin{aligned}
& R_{j}^{\epsilon}(\lambda)=\frac{1}{\sqrt{\pi}} \int_{\epsilon<|r|<1 / \epsilon} \int_{\mathbb{C}^{n}}\left(-\frac{\partial}{\partial \xi_{j}}+\lambda \xi_{j}\right) \pi_{\lambda}(r z) q_{1}\left(z,-\lambda r^{2}\right) d z d r, \\
& R_{j}^{* \epsilon}(\lambda)=\frac{1}{\sqrt{\pi}} \int_{\epsilon<|r|<1 / \epsilon} \int_{\mathbb{C}^{n}}\left(\frac{\partial}{\partial \xi_{j}}+\lambda \xi_{j}\right) \pi_{\lambda}(r z) q_{1}\left(z,-\lambda r^{2}\right) d z d r .
\end{aligned}
$$


Now using the previous lemma,

$$
\begin{aligned}
i r & \int_{\mathbb{C}^{n}} \lambda \xi_{j} \pi_{\lambda}(r z) q_{1}\left(\frac{z}{r},-\lambda r^{2}\right) d z \\
& =\int_{\mathbb{C}^{n}}\left(\frac{\partial}{\partial x_{j}}-\frac{i \lambda r^{2} y_{j}}{2}\right) \pi_{\lambda}(r z) q_{1}\left(z,-\lambda r^{2}\right) d z \\
& =-\int_{\mathbb{C}^{n}} \pi_{\lambda}(r z)\left(\frac{\partial}{\partial x_{j}}+\frac{i \lambda r^{2} y_{j}}{2}\right) q_{1}\left(z,-\lambda r^{2}\right) d z \\
& =-\int_{\mathbb{C}^{n}} \pi_{\lambda}(r z)\left(\frac{\partial}{\partial x_{j}}+\frac{i \lambda r^{2} y_{j}}{2}\right)\left(\int_{\mathbb{R}} p_{1}(z, t) e^{i \lambda r^{2} t} d t\right) d z \\
& =-\int_{\mathbb{C}^{n}} \pi_{\lambda}(r z)\left(\int_{\mathbb{R}}\left(\frac{\partial}{\partial x_{j}}-\frac{y_{j}}{2} \frac{\partial}{\partial t}\right) p_{1}(z, t) e^{i \lambda r^{2} t} d t\right) d z \\
& =-\int_{\mathbb{H}^{n}} \pi_{\lambda}(r z) \tilde{X}_{j} p_{1}(z, t) e^{i \lambda r^{2} t} d z d t .
\end{aligned}
$$

Similarly,

$$
\begin{aligned}
r & \int_{\mathbb{C}^{n}}\left(\frac{\partial}{\partial \xi_{j}} \pi_{\lambda}(r z)\right) q_{1}\left(\frac{z}{r},-\lambda r^{2}\right) d z \\
& =\int_{\mathbb{C}^{n}}\left(\frac{\partial}{\partial y_{j}}+\frac{i \lambda r^{2} x_{j}}{2}\right) \pi_{\lambda}(r z) q_{1}\left(z,-\lambda r^{2}\right) d z \\
& =-\int_{\mathbb{H}^{n}} \pi_{\lambda}(r z) \tilde{Y}_{j} p_{1}(z, t) e^{i \lambda r^{2} t} d z d t
\end{aligned}
$$

Hence

$$
R_{j}^{\epsilon}(\lambda)=\frac{1}{\sqrt{\pi}} \int_{\epsilon<|r|<1 / \epsilon} \int_{\mathbb{H}^{n}} \pi_{\lambda}(r z) \tilde{Z}_{j} p_{1}(z, t) e^{i \lambda r^{2} t} d z d t \frac{d r}{r}
$$

and

$$
R_{j}^{* \epsilon}(\lambda)=\frac{1}{\sqrt{\pi}} \int_{\epsilon<|r|<1 / \epsilon} \int_{\mathbb{H}^{n}} \pi_{\lambda}(r z) \tilde{Z}_{j}^{*} p_{1}(z, t) e^{i \lambda r^{2} t} d z d t \frac{d r}{r} .
$$

Thus

$$
R_{j}^{\epsilon} f(\xi, \eta)=\frac{1}{\sqrt{\pi}} \int_{\mathbb{R}}\left(\int_{\epsilon<r<1 / \epsilon} \int_{\mathbb{H}^{n}} \pi_{\lambda}(r z) f^{\lambda}(\xi) \tilde{Z}_{j} p_{1}(z, t) e^{i \lambda r^{2} t} d z d t \frac{d r}{r}\right) e^{i \lambda \eta} d \lambda .
$$

Now the proposition follows from the fact that

$$
\int_{\mathbb{R}} \pi_{\lambda}(r z) f^{\lambda}(\xi) e^{i \lambda r^{2} t} e^{i \lambda \eta} d \lambda=f\left(\xi+r y, \eta+r x \cdot \xi+r^{2}\left(t+\frac{x \cdot y}{2}\right)\right) .
$$

\section{A transference Result}

In the previous section we obtained a representation for the Riesz transforms as the superposition of certain operators $T_{\epsilon}^{(z, t)}$. To prove the boundedness of the vector of Riesz transforms, using the method of rotations, we need to prove that these operators are bounded uniformly in $(z, t)$. 
Proposition 4.1. For $1<p<\infty$, there exists a constant $C_{p}$ independent of $(z, t) \in \mathbb{H}^{n}, \epsilon>0$ and the dimension $n$ such that

$$
\left\|T_{\epsilon}^{(z, t)} f\right\|_{L^{p}\left(\mathbb{R}^{n+1}\right)} \leq C_{p}\|f\|_{L^{p}\left(\mathbb{R}^{n+1}\right)} .
$$

Proof. This will be proved using Calderón's method of transferring an operator on $L^{p}(X)$, for a measure space $X$, to $L^{p}(G)$ when the group $G$ acts on $X$ by measure preserving transformations. The measure space $\mathbb{R}^{n+1}$, the group $\mathbb{H}^{n}$ and the group action

$$
U(x+i y, t)(\xi, \eta)=\left(\xi-y, \eta-t+\frac{x \cdot y}{2}-x . \xi\right)
$$

are all the same as those used by Ratnakumar and Thangavelu in RT98, and we follow their notation. Accordingly, for $f \in L^{p}\left(\mathbb{R}^{n+1}\right)$ and $(\xi, \eta) \in \mathbb{R}^{n+1}$, we define the transferred function $F_{(\xi, \eta)}$ on $\mathbb{H}^{n}$ as

$$
F_{(\xi, \eta)}(z, t)=f(U(z, t)(\xi, \eta)) .
$$

For $T \in \mathcal{B}\left(L^{p}\left(\mathbb{H}^{n}\right)\right)$, the transferred operator $T_{0}$ on $\mathcal{B}\left(L^{p}\left(\mathbb{R}^{n+1}\right)\right)$ is defined as

$$
T_{0} f(\xi, \eta)=\left(T F_{(\xi, \eta)}\right)(0) .
$$

For a curve $\gamma=\left\{\gamma(t) \in \mathbb{H}^{n}, t \in \mathbb{R}\right\}$ and a function $f$ on $\mathbb{H}^{n}$, the Hilbert transform of $f$ along $\gamma$ is defined as

$$
H_{\gamma} f(w, s)=\int_{\mathbb{R}} f\left((w, s) \gamma(r)^{-1}\right) \frac{d r}{r} .
$$

When $\gamma$ is the curve $\left(r z, r^{2} t\right)$, we will denote $H_{\gamma}$ by $H_{(z, t)}$. By $H_{(z, t)}^{\epsilon}$ we denote the truncated Hilbert transform where the integration is only over $\{r \in \mathbb{R}, \epsilon<|r|<$ $1 / \epsilon\}$. When we transfer this operator $H_{(z, t)}^{\epsilon}$ to an operator on $L^{p}\left(\mathbb{R}^{n+1}\right)$ under the $U$ action defined above, we get

$$
\begin{aligned}
\left(H_{(z, t)}^{\epsilon}\right)_{0} f(\xi, \eta) & =H_{(z, t)}^{\epsilon} F_{(\xi, \eta)}(0)=\int_{\epsilon<|r|<1 / \epsilon} F_{(\xi, \eta)}\left(-r z,-r^{2} t\right) \frac{d r}{r} \\
& =\int_{\epsilon<|r|<1 / \epsilon} f\left(\xi+r y, \eta+r(x \cdot \xi)+r^{2}\left(t+\frac{x \cdot y}{2}\right)\right) \frac{d r}{r} .
\end{aligned}
$$

Hence we see that $\left(H_{(z, t)}^{\epsilon}\right)_{0}=T_{\epsilon}^{(z, t)}$. Now our aim is to prove

$$
\left\|T_{\epsilon}^{(z, t)} f\right\|_{L^{p}\left(\mathbb{R}^{n+1}\right)} \leq C_{p}\|f\|_{L^{p}\left(\mathbb{R}^{n+1}\right)} .
$$

This can be obtained using the transference method from the uniform boundedness of the operator $H_{(z, t)}^{\epsilon}$. To be precise, we use the fact that there exist a finite constant $C_{p}$, independent of $(z, t) \in \mathbb{H}^{n}, n$ and $\epsilon>0$ such that

$$
\left\|H_{(z, t)}^{\epsilon} f\right\|_{p} \leq C_{p}\|f\|_{p} .
$$

A proof of the above fact is indicated in CMZ96. We will present it at the end of this section after proving the boundedness of $T_{\epsilon}^{(z, t)}$. Then

$$
\begin{aligned}
\int_{\mathbb{R}^{n+1}}\left|T_{\epsilon}^{(z, t)} f(\xi, \eta)\right|^{p} d \xi d \eta & =\int_{\mathbb{R}^{n+1}}\left|H_{(z, t)}^{\epsilon} F_{(\xi, \eta)}(0)\right|^{p} d \xi d \eta \\
& =\int_{\mathbb{R}^{n+1}}\left|H_{(z, t)}^{\epsilon} F_{U(w, s)(\xi, \eta)}(0)\right|^{p} d \xi d \eta
\end{aligned}
$$


because of the invariance of the measure $d \xi d \eta$ under the $U$ action. Hence

$$
\begin{aligned}
\int_{\mathbb{R}^{n+1}}\left|T_{\epsilon}^{(z, t)} f(\xi, \eta)\right|^{p} d \xi d \eta & =\frac{1}{\left|B_{R}(0)\right|} \int_{B_{R}(0)} \int_{\mathbb{R}^{n+1}}\left|H_{(z, t)}^{\epsilon} F_{U(w, s)(\xi, \eta)}(0)\right|^{p} d \xi d \eta d w d s \\
& =\frac{1}{\left|B_{R}(0)\right|} \int_{B_{R}(0)} \int_{\mathbb{R}^{n+1}}\left|H_{(z, t)}^{\epsilon} F_{(\xi, \eta)}(w, s)\right|^{p} d \xi d \eta d w d s
\end{aligned}
$$

where $B_{R}(0)$ is the ball centred at the origin in the Heisenberg group and radius $R$ under the Koranyi norm $\|(w, s)\|=\left(|w|^{4}+|s|^{2}\right)^{1 / 4}$. When $(w, s) \in B_{R}(0)$ and $\epsilon<|r|<1 / \epsilon,(w, s)\left(\delta_{r}(z, t)\right)^{-1} \in B_{R+\frac{h}{\epsilon}}(0)$, where $h$ is the Koranyi norm of $(z, t)$. Hence, in the above equality $F_{(\xi, \eta)}$ can be replaced by $\tilde{F}_{(\xi, \eta)}=F_{(\xi, \eta)} \cdot \chi_{B_{R+h / \epsilon}}(0)$. Now by an application of Fubini, we get

$$
\left\|T_{\epsilon}^{(z, t)} f\right\|_{p}^{p} \leq \frac{1}{\left|B_{R}(0)\right|} \int_{\mathbb{R}^{n+1}} \int_{\mathbb{H}^{n}}\left|H_{(z, t)}^{\epsilon} \tilde{F}_{(\xi, \eta)}(w, s)\right|^{p} d w d s d \xi d \eta .
$$

Now, from the uniform boundedness of the truncated Hilbert transforms, we get

$$
\begin{aligned}
\left\|T_{\epsilon}^{(z, t)} f\right\|_{p}^{p} & \leq C_{p} \frac{1}{\left|B_{R}(0)\right|} \int_{\mathbb{R}^{n+1}} \int_{\mathbb{H}^{n}}\left|\tilde{F}_{(\xi, \eta)}(w, s)\right|^{p} d w d s d \xi d \eta \\
& =C_{p} \frac{1}{\left|B_{R}(0)\right|} \int_{B_{R+h / \epsilon}(0)} \int_{\mathbb{R}^{n+1}}|f(U(w, s)(\xi, \eta))|^{p} d \xi d \eta d w d s \\
& =C_{p} \frac{\left|B_{R+h / \epsilon}(0)\right|}{\left|B_{R}(0)\right|}\|f\|_{p}^{p}=C_{p}\left(\frac{R+h / \epsilon}{R}\right)^{2 n+2}\|f\|_{p}^{p},
\end{aligned}
$$

again by the invariance of $d \xi d \eta$ under the $U$ action. Letting $R \rightarrow \infty$, we see that

$$
\left\|T_{\epsilon}^{(z, t)} f\right\|_{L^{p}\left(\mathbb{R}^{n}\right)} \leq C_{p}\|f\|_{L^{p}\left(\mathbb{R}^{n}\right)} .
$$

Coming back to the boundedness of $H_{(z, t)}^{\epsilon}$, we can use a technique in Lemma 3.1 of [Str91] to reduce this to the boundedness of $H_{\gamma}^{\epsilon}$ on $L^{p}\left(\mathbb{R}^{2}\right)$ for the curve $\gamma=$ $\left\{\left(t, t^{2}\right), t \in \mathbb{R}\right\}$. Recall that

$$
H_{(z, t)} f(w, s)=\int_{\mathbb{R}} f\left(w-r z, s-r^{2} t-r \operatorname{Im}(w \cdot \bar{z})\right) \frac{d r}{r} .
$$

For $\sigma \in U(n)$, define $\rho(\sigma) f(w, s)=f(\sigma w, s)$. Then

$$
\begin{aligned}
H_{(z, t)}(\rho(\sigma) f)(w, s) & =\int_{\mathbb{R}} f\left(\sigma w-r \sigma z, s-r^{2} t-r \operatorname{Im}(w \cdot \bar{z})\right) \frac{d r}{r} \\
\rho\left(\sigma^{-1}\right) H_{(z, t)}(\rho(\sigma) f)(w, s) & =H_{(z, t)}(\rho(\sigma) f)\left(\sigma^{-1} w, s\right) \\
& =\int_{\mathbb{R}} f\left(w-r \sigma z, s-r^{2} t-r \operatorname{Im}\left(\sigma^{-1} w \cdot \bar{z}\right)\right) \frac{d r}{r} \\
& =\int_{\mathbb{R}} f(w-r \sigma z, s-t-r \operatorname{Im}(w \cdot \bar{\sigma} z)) \frac{d r}{r} .
\end{aligned}
$$

Since $\|\rho(\sigma) f\|_{p}=\|f\|_{p}$ and since there exists $\sigma \in U(n)$ such that $\rho(\sigma)(x+i y)=$ $x_{1} e_{1}$, it is enough to consider the operator

$$
f \rightarrow \int_{\mathbb{R}} f\left(u_{1}-r x_{1}, w^{\prime}, s-r^{2} t-r v_{1} x_{1}\right) \frac{d r}{r},
$$


which is equivalent to the operator

$$
T_{(x, t, v)} f(u, s)=\int_{\mathbb{R}} f\left(u-r x, s-r^{2} t-r v x\right) \frac{d r}{r}
$$

acting on functions defined on $\mathbb{R}^{2}$. For $\lambda_{1}, \lambda_{2}>0$, let

$$
\delta_{\lambda_{1}, \lambda_{2}} f(u, s)=f\left(\lambda_{1} u, \lambda_{2} s\right) .
$$

Then $\left\|\delta_{\lambda_{1}, \lambda_{2}} f\right\|_{p}=\left(\lambda_{1} \lambda_{2}\right)^{-1 / p}\|f\|_{p}$ and

$$
\delta_{1 / \lambda_{1}, 1 / \lambda_{2}} T_{(x, t, v)} \delta_{\lambda_{1}, \lambda_{2}} f(u, s)=\int_{\mathbb{R}} f\left(u-r \lambda_{1} x, s-r^{2} \lambda_{2} t-r \lambda_{2} v x\right) \frac{d r}{r} .
$$

Since we can choose $\lambda_{1}, \lambda_{2}$ such that $\lambda_{1} x=\lambda_{2} t=1$, it is enough to get uniform estimates for operators of the form $T_{(1,1, a)}$. That would imply that

$$
\begin{aligned}
\left\|T_{(x, t, v)} f\right\|_{p} & =\left\|\delta_{\lambda_{1}, \lambda_{2}} T_{(1,1, v x / t)} \delta_{1 / \lambda_{1}, 1 / \lambda_{2}} f\right\|_{p} \\
& =\left(\lambda_{1} \lambda_{2}\right)^{-1 / p}\left\|T_{(1,1, v x / t)} \delta_{1 / \lambda_{1}, 1 / \lambda_{2}} f\right\|_{p} \\
& \leq\left(\lambda_{1} \lambda_{2}\right)^{-1 / p} C_{p}\left\|\delta_{1 / \lambda_{1}, 1 / \lambda_{2}} f\right\|_{p} \\
& =\left(\lambda_{1} \lambda_{2}\right)^{-1 / p} C_{p}\left(\lambda_{1} \lambda_{2}\right)^{1 / p}\|f\|_{p} .
\end{aligned}
$$

Now consider $\tau_{a} f(u, s)=f(u, s+a u)$ so that $\left\|\tau_{a} f\right\|_{p}=\|f\|_{p}$. Since

$$
\begin{aligned}
T_{(1,1, a)}\left(\tau_{a}^{-1} f\right)(u, s) & =\int_{\mathbb{R}}\left(\tau_{a}^{-1} f\right)\left(u-r, s-r^{2}-a r\right) \frac{d r}{r} \\
& =\int_{\mathbb{R}} f\left(u-r, s-a u-r^{2}\right) \frac{d r}{r},
\end{aligned}
$$

we have

$$
\begin{aligned}
\tau_{a} T_{(1,1, a)}\left(\tau_{a}^{-1} f\right)(u, s) & =T_{(1,1, a)}\left(\tau_{a}^{-1} f\right)(u, s+a u) \\
& =\int_{\mathbb{R}} f\left(u-r, s-r^{2}\right) \frac{d r}{r} .
\end{aligned}
$$

This is the Hilbert transform along the parabola $\gamma(r)=\left(r, r^{2}\right)$ in $\mathbb{R}^{2}$. Since

$$
\gamma(r)= \begin{cases}\delta_{r}(1,1) & \text { if } r>0 \\ 0 & \text { if } r=0 \\ \delta_{-r}(-1,1) & \text { if } r<0\end{cases}
$$

and the linear space spanned by $\{\gamma(r)\}_{r>0}$ is the same as the linear space spanned by $\{\gamma(r)\}_{r<0}$, namely $\mathbb{R}^{2}, \gamma(r)$ is a two-sided homogeneous curve (see Definition 3.1 in [SW78, p. 1261]. Now we can appeal to Theorem 11 of [SW78, p. 1271] to get the uniform boundedness of the truncated Hilbert transforms $H_{(z, t)}^{\epsilon}$ on $L^{p}\left(\mathbb{H}^{n}\right)$. That is, there exist a finite constant $C_{p}$, independent of $(z, t) \in \mathbb{H}^{n}, n$ and $\epsilon>0$, such that

$$
\left\|H_{(z, t)}^{\epsilon} f\right\|_{p} \leq C_{p}\|f\|_{p}
$$




\section{Proof OF the MAIN THEOREM}

We first prove Theorem 2.2 regarding the boundedness of the individual Riesz transforms $R_{j}$ and $R_{j}^{*}$.

Proof of Theorem 2.2. We first consider the operators $R_{j}^{\epsilon}$ and $R_{j}^{* \epsilon}$. In light of Propositions 3.1 and 4.1 , we just need to prove that there exist a finite $C$, independent of $n$, such that

$$
\left\|\tilde{Z}_{j} p_{1}(z, t)\right\|_{L^{1}\left(\mathbb{H}^{n}\right)},\left\|\tilde{Z}_{j}^{*} p_{1}(z, t)\right\|_{L^{1}\left(\mathbb{H}^{n}\right)} \leq C .
$$

As mentioned in Lemma 3 of CMZ96, this follows from the fact that

$$
p_{r}^{n}\left(z_{1}, z_{2}, \cdots, z_{n}, t\right)=p_{r}^{1}\left(z_{1}, \cdot\right) * p_{r}^{1}\left(z_{2}, \cdot\right), \cdots, * p_{r}^{1}\left(z_{n}, \cdot\right)(t),
$$

where $p_{r}^{n}$ is the heat kernel on $\mathbb{H}^{n}$. Now for the operators $R_{j}$ and $R_{j}^{*}$, we need only to show that $\left(R_{j}^{\epsilon} f\right),\left(R_{j}^{* \epsilon} f\right)$ are Cauchy in $L^{p}\left(\mathbb{R}^{n+1}\right)$. We have already seen that $\left\|R_{j}^{\epsilon} f\right\|_{p} \leq C\left\|T_{\epsilon}^{(z, t)} f\right\|_{p}$, and so it is enough to prove that $T_{\epsilon}^{(z, t)} f$ is Cauchy in $L^{p}\left(\mathbb{R}^{n+1}\right)$. During the course of the proof of Proposition 4.1, we had observed that the operator $T_{\epsilon}^{(z, t)}$ is obtained by applying a transference on $H_{(z, t)}^{\epsilon}$ and that

$$
\left\|T_{\epsilon}^{(z, t)} f\right\|_{L^{p}\left(\mathbb{R}^{n+1}\right)} \leq\left\|H_{(z, t)}^{\epsilon} f\right\|_{L^{p}\left(\mathbb{H}^{n}\right)} .
$$

Being truncated Hilbert transforms, $H_{(z, t)}^{\epsilon} f$ is Cauchy in $L^{p}\left(\mathbb{H}^{n}\right)$ SW78, Theorem 11], and consequently, so is $T_{\epsilon}^{(z, t)} f$.

Now we complete the proof of our main theorem. As mentioned during the discussion of Theorem 2.2, we only need to prove the boundedness of the operator $\mathcal{R}^{\epsilon}$ obtained by replacing $R_{j}$ and $R_{j}^{*}$ by their truncated versions. We will be closely following [LP04] in proving this and so will skip some details. Using the property

$$
\frac{1}{r} \frac{\partial p_{1}}{\partial r}=\frac{1}{x_{j}} \frac{\partial p_{1}}{\partial x_{j}}=\frac{1}{y_{j}} \frac{\partial p_{1}}{\partial y_{j}}
$$

where $r=|z|$, we can rewrite

$$
\begin{aligned}
R_{j}^{\epsilon} f(\xi, \eta)=\frac{1}{\sqrt{\pi}} \int_{\mathbb{H}^{n}}\left(i x_{j}+y_{j}\right) T_{\epsilon}^{(z, t)} f(\xi, \eta) \frac{1}{r} \frac{\partial p_{1}}{\partial r}(z, t) d z d t \\
+\frac{1}{2 \sqrt{\pi}} \int_{\mathbb{H}^{n}}\left(x_{j}-y_{j}\right) T_{\epsilon}^{(z, t)} f(\xi, \eta) \frac{\partial p_{1}}{\partial t}(z, t) d z d t
\end{aligned}
$$

and

$$
\begin{aligned}
R_{j}^{* \epsilon} f(\xi, \eta)=\frac{1}{\sqrt{\pi}} \int_{\mathbb{H}^{n}}\left(i x_{j}-y_{j}\right) & T_{\epsilon}^{(z, t)} f(\xi, \eta) \frac{1}{r} \frac{\partial p_{1}}{\partial r}(z, t) d z d t \\
& -\frac{1}{2 \sqrt{\pi}} \int_{\mathbb{H}^{n}}\left(x_{j}+y_{j}\right) T_{\epsilon}^{(z, t)} f(\xi, \eta) \frac{\partial p_{1}}{\partial t}(z, t) d z d t
\end{aligned}
$$

For a fixed $(\xi, \eta)$ we can choose $\lambda_{1}, \lambda_{2}, \ldots, \lambda_{2 n}$ such that $\sum_{j=1}^{2 n}\left|\lambda_{j}\right|^{2}=1$ and

$$
\left|\mathcal{R}^{\epsilon} f(\xi, \eta)\right|=\sum_{j=1}^{n}\left(\lambda_{j} \overline{R_{j}^{\epsilon} f(\xi, \eta)}+\lambda_{j+n} \overline{R_{j}^{* \epsilon} f(\xi, \eta)}\right) .
$$


Now using the triangle inequality, Hölder's inequality and also Lemma 2(a) of [LP04, we get

$$
\begin{aligned}
\left|\mathcal{R}^{\epsilon} f(\xi, \eta)\right| \leq C\left\|T_{\epsilon}^{(z, t)} f(\xi, \eta)\right\|_{L^{p}\left(\frac{1}{r} \frac{\partial p_{1}}{\partial r} d z d t\right)}\left\|x_{1}\right\|_{L^{p^{\prime}}\left(\frac{1}{r} \frac{\partial p_{1}}{\partial r} d z d t\right)} & \\
& +C\left\|T_{\epsilon}^{(z, t)} f(\xi, \eta)\right\|_{L^{p}\left(\frac{\partial p_{1}}{\partial t} d z d t\right)}\left\|x_{1}\right\|_{L^{p^{\prime}}\left(\frac{\partial p_{1}}{\partial t} d z d t\right)}
\end{aligned}
$$

where $C$ is a universal constant. Now the theorem follows from Proposition 4.1 and an application of Minkowski inequality along with the fact (proved in [LP04]) that

$$
\int_{\mathbb{H}^{n}}\left|x_{1}\right|^{p} \frac{1}{r} \frac{\partial p_{1}}{\partial r} d z d t, \quad \int_{\mathbb{H}^{n}}\left|x_{1}\right|^{p} \frac{\partial p_{1}}{\partial t} d z d t \leq A_{p}, p \geq 0
$$

where $A_{p}$ is independent of the dimension.

\section{ACKNOWLEDGMENTS}

The authors are thankful to the referee for pointing out some errors and typos. The work of the first author was supported by the All India Council for Technical Education (AICTE). The work of the second author was supported by a J. C. Bose Fellowship from the Department of Science and Technology (DST) and also by a grant from UGC via DSA-SAP.

\section{REFERENCES}

[BG] F. Baudoin and N. Garofalo, A note on the boundedness of Riesz transform for some subelliptic operators, Int. Math. Res. Not. IMRN 2013, no. 2, 398-421. MR3010694

[CMZ96] T. Coulhon, D. Müller, and J. Zienkiewicz, About Riesz transforms on the Heisenberg groups, Math. Ann. 305 (1996), no. 2, 369-379. MR.1391221 (97f:22015)

[dL65] Karel de Leeuw, On $L_{p}$ multipliers, Ann. of Math. (2) 81 (1965), 364-379. MR0174937 (30 \#5127)

[DR85] J. Duoandikoetxea and José L. Rubio de Francia, Estimations indépendantes de la dimension pour les transformées de Riesz, C. R. Acad. Sci. Paris Sér. I Math. 300 (1985), no. 7, 193-196. MR780616 (86e:42028)

[HRST04] E. Harboure, L. de Rosa, C. Segovia, and J. L. Torrea, $L^{p}$-dimension free boundedness for Riesz transforms associated to Hermite functions, Math. Ann. 328 (2004), no. 4, 653-682. MR2047645 (2006a:42017)

[JST] K. Jotsaroop, P. K. Sanjay, and S. Thangavelu, Riesz transforms and multipliers for the Grushin operator, J. Anal. Math. 119 (2013), 255-273. MR3043153

[LP04] Françoise Lust-Piquard, Riesz transforms on generalized Heisenberg groups and Riesz transforms associated to the CCR heat flow, Publ. Mat. 48 (2004), no. 2, 309-333, DOI 10.5565/PUBLMAT_48204_02. MR2091008(2005g:43014)

[LP06] Françoise Lust-Piquard, Dimension free estimates for Riesz transforms associated to the harmonic oscillator on $\mathbb{R}^{n}$, Potential Anal. 24 (2006), no. 1, 47-62, DOI 10.1007/s11118-005-4389-1. MR2218202(2006k:42012)

[Pis88] Gilles Pisier, Riesz transforms: a simpler analytic proof of P.-A. Meyer's inequality, Séminaire de Probabilités, XXII, Lecture Notes in Math., vol. 1321, Springer, Berlin, 1988, pp. 485-501, DOI 10.1007/BFb0084154. MR960544(89m:60178)

[RT98] P. K. Ratnakumar and S. Thangavelu, Spherical means, wave equations, and Hermite-Laguerre expansions, J. Funct. Anal. 154 (1998), no. 2, 253-290, DOI 10.1006/jfan.1997.3135. MR1612697 (99h:33047)

[Ste83] E. M. Stein, Some results in harmonic analysis in $\mathbf{R}^{n}$, for $n \rightarrow \infty$, Bull. Amer. Math. Soc. (N.S.) $\mathbf{9}$ (1983), no. 1, 71-73. MR699317 (84g:42019)

[Str91] R. S. Strichartz, $L^{p}$ harmonic analysis and Radon transforms on the Heisenberg group, J. Funct. Anal. 96 (1991), no. 2, 350-406. MR.1101262 (92d:22015) 
[ST12] P. K. Sanjay and Sundaram Thangavelu, Revisiting Riesz transforms on Heisenberg groups, Rev. Mat. Iberoam. 28 (2012), no. 4, 1091-1108, DOI 10.4171/RMI/704. MR2990135

[SW78] Elias M. Stein and Stephen Wainger, Problems in harmonic analysis related to curvature, Bull. Amer. Math. Soc. 84 (1978), no. 6, 1239-1295, DOI 10.1090/S0002-99041978-14554-6. MR.508453 (80k:42023)

[Tha04] Sundaram Thangavelu, An introduction to the uncertainty principle: Hardy's theorem on Lie groups, with a foreword by Gerald B. Folland. Progress in Mathematics, vol. 217, Birkhäuser Boston Inc., Boston, MA, 2004. MR2008480 (2004j:43007)

[Wei01] Lutz Weis, Operator-valued Fourier multiplier theorems and maximal $L_{p}$-regularity, Math. Ann. 319 (2001), no. 4, 735-758, DOI 10.1007/PL00004457. MR.1825406 (2002c:42016) INDIA

Department of Mathematics, National Institute of Technology, Calicut 673601 ,

Current address: Department of Mathematics, Indian Institute of Science, Bangalore 560012 , India

E-mail address: sanjay@math.iisc.ernet.in

Department of Mathematics, Indian Institute of Science, Bangalore 560 012, India

E-mail address: veluma@math.iisc.ernet.in 